\title{
ALMA OBSERVATIONS OF THE ORION PROPLYDS
}

\author{
Rita K. Mann $^{1}$, James Di Francesco ${ }^{1,2}$, Doug Johnstone ${ }^{1,2,3}$, Sean M. Andrews ${ }^{4}$, Jonathan P. Williams ${ }^{5}$, \\ John Bally ${ }^{6}$, LuCa Ricci $^{7}$, A. Meredith Hughes ${ }^{8}$, and Brenda C. Matthews ${ }^{1,2}$ \\ ${ }^{1}$ National Research Council Canada, 5071 West Saanich Road, Victoria, BC, V9E 2E7, Canada; rita.mann@ nrc-cnrc.gc.ca \\ ${ }^{2}$ Department of Physics and Astronomy, University of Victoria, Victoria, BC, V8P 1A1, Canada \\ 3 Joint Astronomy Centre, 660 North A'ohoku Place, University Park, Hilo, HI 96720, USA \\ ${ }^{4}$ Harvard-Smithsonian Center for Astrophysics, 60 Garden Street, Cambridge, MA 02138, USA \\ ${ }^{5}$ Institute for Astronomy, University of Hawaii, 2680 Woodlawn Drive, Honolulu, HI 96822 USA \\ ${ }^{6}$ CASA, University of Colorado, CB 389, Boulder, CO 80309, USA \\ ${ }^{7}$ Department of Astronomy, California Institute of Technology, MC 249-17, Pasadena, CA 91125, USA \\ ${ }^{8}$ Van Vleck Observatory, Astronomy Department, Wesleyan University, 96 Foss Hill Drive, Middletown, CT 06459, USA \\ Received 2013 October 31; accepted 2014 February 11; published 2014 March 6
}

\begin{abstract}
We present Atacama Large Millimeter/submillimeter Array (ALMA) observations of protoplanetary disks ("proplyds") in the Orion Nebula Cluster. We imaged five individual fields at $856 \mu \mathrm{m}$ containing 22 Hubble Space Telescope (HST)-identified proplyds and detected 21 of them. Eight of those disks were detected for the first time at submillimeter wavelengths, including the most prominent, well-known proplyd in the entire Orion Nebula, 114-426. Thermal dust emission in excess of any free-free component was measured in all but one of the detected disks, and ranged between 1 and $163 \mathrm{mJy}$, with resulting disk masses of 0.3-79 $M_{\text {jup }}$. An additional 26 stars with no prior evidence of associated disks in HST observations were also imaged within the 5 fields, but only 2 were detected. The disk mass upper limits for the undetected targets, which include OB stars, $\theta^{1}$ Ori $\mathrm{C}$, and $\theta^{1}$ Ori F, range from 0.1 to $0.6 M_{\text {jup }}$. Combining these ALMA data with previous Submillimeter Array observations, we find a lack of massive ( $\gtrsim 3 M_{\text {jup }}$ ) disks in the extreme-UV-dominated region of Orion, within $0.03 \mathrm{pc}$ of $\theta^{1}$ Ori C. At larger separations from $\theta^{1}$ Ori $\mathrm{C}$, in the far-UV-dominated region, there is a wide range of disk masses, similar to what is found in low-mass star forming regions. Taken together, these results suggest that a rapid dissipation of disk masses likely inhibits potential planet formation in the extreme-UV-dominated regions of OB associations, but leaves disks in the far-UV-dominated regions relatively unaffected.
\end{abstract}

Key words: protoplanetary disks - submillimeter: planetary systems

Online-only material: color figures

\section{INTRODUCTION}

Circumstellar disks are the birthsites of exoplanets. Hubble Space Telescope (HST) images of the Orion Nebula Cluster (ONC) revealed a hostile environment; many of the disks that orbit low mass stars are being photoevaporated by the intense UV radiation from the most massive nearby star, $\theta^{1}$ Ori $\mathrm{C}$ (spectral type O6; O'Dell \& Wen 1994; McCullough et al. 1995; Bally et al. 1998a; Smith et al. 2005; Ricci et al. 2008). These disks are surrounded by tear-drop-shaped structures with bright heads facing $\theta^{1}$ Ori $\mathrm{C}$ and tails pointing radially away. These distinctive circumstellar morphologies led to the nomenclature "proplyds," an acronym for protoplanetary disks, that is now regularly applied to low-mass stars and their disks in the centers of massive star forming regions (O’Dell \& Wen 1994). The Orion proplyds were found to suffer photoevaporative massloss rates of $\dot{M} \approx 10^{-7} M_{\odot} \mathrm{yr}^{-1}$ (Churchwell et al. 1987; Henney \& O'Dell 1999), high enough to disperse the amount of disk mass required to form a planetary system like our own in under 1 Myr. That dissipation timescale is too short compared to the core accretion requirements for giant planet formation (e.g., Hubickyj et al. 2005), and is in apparent conflict with the inferred ages of the ONC stars ( 2 Myr; Reggiani et al. 2011; Da Rio et al. 2009).

Measurements of the masses that remain in the Orion proplyds are crucial for characterizing the photoevaporation process and assessing their potential for planet formation. The most straightforward way to estimate a disk mass is from a measurement of the thermal dust continuum luminosity at long wavelengths, where the emission is optically thin (cf. Beckwith et al. 1990). Although molecular gas likely comprises the vast majority of the mass budget in disks, the dust dominates the opacity and is significantly easier to detect. The Berkeley-Illinois-Maryland Array, Owens Valley Radio Observatory, Plateau de Bure Interferometer, and Combined Array for Research in Millimeter Astronomy observed the Orion proplyds at wavelengths of 1.3-3.5 mm (Mundy et al. 1995; Bally et al. 1998b; Lada 1998; Eisner \& Carpenter 2006; Eisner et al. 2008), but unfortunately these data provided limited constraints on the disk masses: contamination by free-free radiation from the ionized cocoons generated by the photoevaporation process often dominated the dust emission. Soon after the Submillimeter Array (SMA) was commissioned, it produced the first successful detections of the Orion proplyds at a submillimeter wavelength $(880 \mu \mathrm{m})$, where the dust emission dominates (Williams et al. 2005). Those observations revealed that at least some of these disks still have sufficient mass ( $>10 M_{\text {jup }}$ ) remaining to potentially form giant planets. A larger scale SMA survey of the Orion proplyds identified the erosion of the high end of the disk mass distribution due to photoevaporation by $\theta^{1}$ Ori C (Mann \& Williams 2009a, 2010). To date, however, these surveys have only been sensitive enough to detect the most massive disks $\left(\geqslant 8.4 M_{\text {jup }}\right)$ in the ONC, and therefore provide relatively biased information about disk evolution in the hearts of massive star forming regions.

To probe the full disk mass distribution in the ONC and further study the impact of external photoionizing radiation on disk properties, we carried out a much more sensitive 
Table 1

Summary of the ALMA Early Science Observations

\begin{tabular}{lcccccc}
\hline \hline Field & $\begin{array}{c}\alpha(\mathrm{J} 2000) \\
(\mathrm{h} \mathrm{m} \mathrm{s})\end{array}$ & $\begin{array}{c}\delta(\mathrm{J} 2000) \\
(\mathrm{deg} \mathrm{m} \mathrm{s})\end{array}$ & $\begin{array}{c}\text { Integration Time } \\
(\mathrm{sec})\end{array}$ & $\begin{array}{c}1 \sigma \mathrm{rms} \\
\left(\mathrm{mJy} \mathrm{beam}^{-1}\right)\end{array}$ & $\begin{array}{c}\text { Beam } \\
\left({ }^{\prime \prime}\right)\end{array}$ & $\begin{array}{c}\text { P.A. } \\
\left({ }^{\circ}\right)\end{array}$ \\
\hline 1 & $05: 35: 16.30$ & $-05: 23: 22.40$ & 2600 & 0.24 & $0.50 \times 0.44$ & 84 \\
2 & $05: 35: 17.35$ & $-05: 23: 38.50$ & 1300 & 0.24 & $0.51 \times 0.46$ & 86 \\
3 & $05: 35: 11.40$ & $-05: 24: 24.00$ & 1300 & $0.58^{\mathrm{a}}$ & $0.51 \times 0.46$ & 88 \\
4 & $05: 35: 25.30$ & $-05: 15: 35.50$ & 1300 & 1.15 & $0.51 \times 0.46$ & 89 \\
5 & $05: 35: 21.50$ & $-05: 09: 42.00$ & 1300 & 0.41 & $0.51 \times 0.46$ & 89 \\
\hline
\end{tabular}

Notes. ${ }^{\text {a }}$ The rms value of Field 3 is itself uncertain by a factor of $\sim 2$ due to the presence of extensive low-level emission in that field.

pilot survey with the Atacama Large Millimeter/submillimeter Array (ALMA) that targeted 48 young stars, including 22 HSTidentified proplyds. In this article, we present the results of the $856 \mu \mathrm{m}$ (ALMA Band 7) continuum observations. The observations and data reduction are described in Section 2. Estimates of disk masses are presented in Section 3, and an examination of the dependence of disk mass on location in the ONC is discussed in Section 4.

\section{OBSERVATIONS}

Five individual pointings (hereafter Fields) in the ONC were observed with ALMA using the Band 7 (345 GHz) receivers on 2012 October 24, as part of the Cycle 0 Early Science operations (see Table 1). Figure 1 marks the pointing centers, with reference to the high mass members of the ONC. Twentytwo $12 \mathrm{~m}$ diameter ALMA antennas were arranged in a hybrid configuration that yielded (with robust weighting) images with a $\sim 0^{\prime \prime} .5$ angular resolution. This target resolution was chosen to distinguish individual disks toward the crowded Trapezium cluster, resolve the emission from three large proplyds, and filter out potentially confusing large-scale emission from the background molecular cloud. Each Field was observed for $136 \mathrm{~s}$ per visit. Fields $2-5$ were visited six times over $6.5 \mathrm{hr}$ to improve sampling in the Fourier plane, but Field 1 was observed twice as often as the others to achieve higher sensitivity for the disks nearest to $\theta^{1}$ Ori $\mathrm{C}$. The correlator was configured to observe simultaneously four $1.875 \mathrm{GHz}$-wide spectral windows, each divided into 3840 channels each with a width of $488.28 \mathrm{kHz}$; after online Hanning smoothing, the spectral resolution was $976.56 \mathrm{kHz}$. The spectral windows were arranged to cover the $\mathrm{CO}(3-2), \mathrm{HCN}(4-3), \mathrm{HCO}^{+}(4-3)$, and CS (7-6) emission lines. The focus here will be on the wideband $(\Delta v \approx 7.5 \mathrm{GHz})$ continuum emission, extracted by integrating over all line-free channels: the spectral line data will be presented elsewhere. At $856 \mu \mathrm{m}$, the mean wavelength of the four spectral windows, the effective field of view is $18^{\prime \prime}$ (the FWHM primary beam of an individual antenna). Since the shortest baseline was $21.2 \mathrm{~m}$ in length, the maximum recoverable scale was 4".99.

Data calibration and image reconstruction were performed using standard procedures in the CASA package. The antennabased complex gains were calibrated based on repeated observations of the quasar J0607-085. The absolute amplitude scale was determined from observations of Callisto, and the bandpass response of the system was measured from observations of the bright quasar J0522-364. The model of Callisto was that provided by Butler (2012). The mean and standard deviation of the $856 \mu \mathrm{m}$ continuum flux of Callisto over all five scheduling blocks and all line-free channels in four spectral windows was $18.52 \mathrm{Jy}$ and $0.53 \mathrm{Jy}$, respectively. Each field was Fourierinverted separately, using the multi-frequency synthesis mode in the CLEAN task with an intermediate Briggs robustness parameter of 0.5 chosen to achieve the desired angular resolution of $\sim 0$. 5 . A dirty image was generated out to a diameter of $\sim 34^{\prime \prime}$ (only $\sim 2 \%$ of the maximum primary beam sensitivity), in an effort to include any bright disks that might have been located at the far margins of each field. These dirty images were then CLEANed with the Clark algorithm down to a threshold of $\sim 2$ times the rms observed in emission-free locations near the pointing centers (boxes were used to focus the algorithm toward features that could be visually confirmed in the dirty images). After CLEANing, each field was corrected for primary beam attenuation and restored with a synthesized $0.51 \times 0.46$ beam. The $1 \sigma$ rms levels obtained toward the center of each Field are listed in Table 1.

\section{RESULTS}

The $856 \mu \mathrm{m}$ continuum maps of the surveyed ONC fields are shown in Figures 2 and 3, alongside the corresponding optical HST images (Bally et al. 2000; Smith et al. 2005). These ALMA images represent the highest resolution observations at submillimeter wavelengths toward the central $\mathrm{OB}$ stars in the ONC. We detected submillimeter emission from 21 of the 22 targeted $H S T$-identified proplyds in this survey; the only proplyd not detected is 169-338 (see Table 2). Of those detections, 8 are new and 13 are recoveries of previous SMA detections. The centroid positions and $856 \mu \mathrm{m}$ integrated flux densities for each target were measured by fitting elliptical Gaussians in the image plane. A suitable rms noise level in each field was determined from the emission-free regions within the primary beam.

The observed emission is composed of a free-free $\left(F_{\mathrm{ff}}\right)$ contribution from the ionized cocoons that surround the photoevaporating disks and the thermal dust emission $\left(F_{\text {dust }}\right)$ from the disks themselves, such that $F_{\text {obs }}=F_{\text {dust }}+F_{\text {ff }}$. The radio-submillimeter spectral energy distributions (SEDs) for the 22 disks detected at $3 \sigma$ at $856 \mu \mathrm{m}$ with ALMA are shown in Figure 4. The free-free contributions from the disk targets were extrapolated from centimeter wavelengths into the submillimeter regime using published Very Large Array flux densities from $1.3 \mathrm{~cm}$ to $6 \mathrm{~cm}$ (Garay et al. 1987; Felli et al. 1993a, 1993b; Zapata et al. 2004). Fits to the free-free emission $\left(F_{\mathrm{ff}} \propto v^{-0.1}\right)$ and dust emission $\left(F_{\text {dust }} \propto v^{2}\right)$ are overlaid on the SEDs to show their relative contributions and contrasting spectral dependences. The radio observations show the flat spectral dependence consistent with optically thin emission, but with a range, highlighted by the gray scale, which we attribute to variability (Felli et al. 1993b; Zapata et al. 2004). We avoided observations taken at wavelengths longer than $6 \mathrm{~cm}(5 \mathrm{GHz})$ in this analysis, in order to avoid the turnover frequency, where the free-free emission becomes optically thick and no longer follows a $v^{-0.1}$ dependence. 


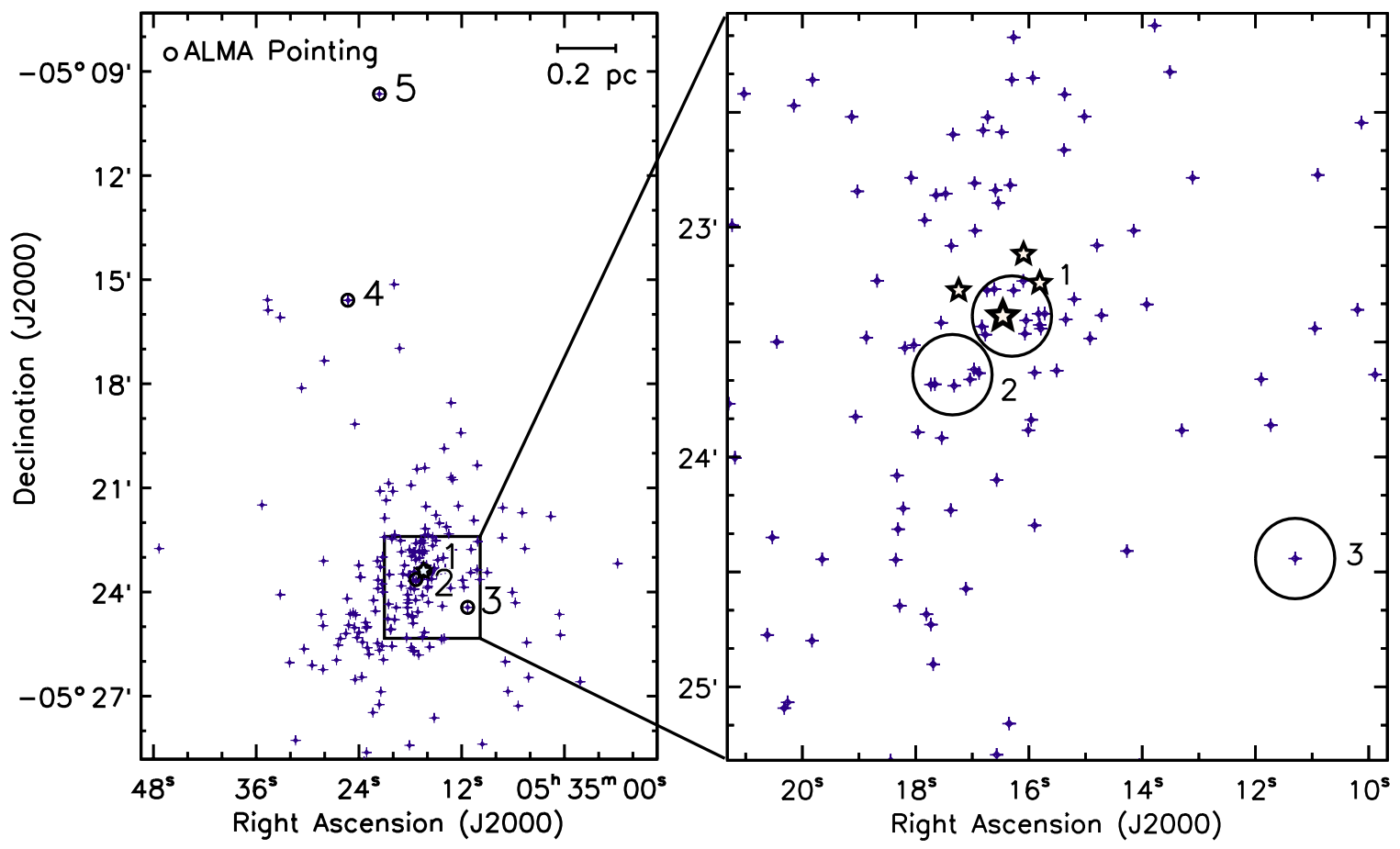

Figure 1. Location of the five observed ALMA fields in the Orion Nebula Cluster. The stars mark the position of the OB stars, and blue crosses show the location of the proplyds identified by $H S T$ observations (Ricci et al. 2008). Black circles represent the $18^{\prime \prime}$ primary beam of the ALMA observations at $856 \mu \mathrm{m}$. Fields $1-5$ are labeled according to Table 1. The black square outlined in the left panel is zoomed in for the panel on the right to allow a better view of the crowded central fields, 1-3, near the OB stars. Fields 1-3 contain 42 of the stars, while Fields 4 and 5 contain the remaining five young stars.

(A color version of this figure is available in the online journal.)
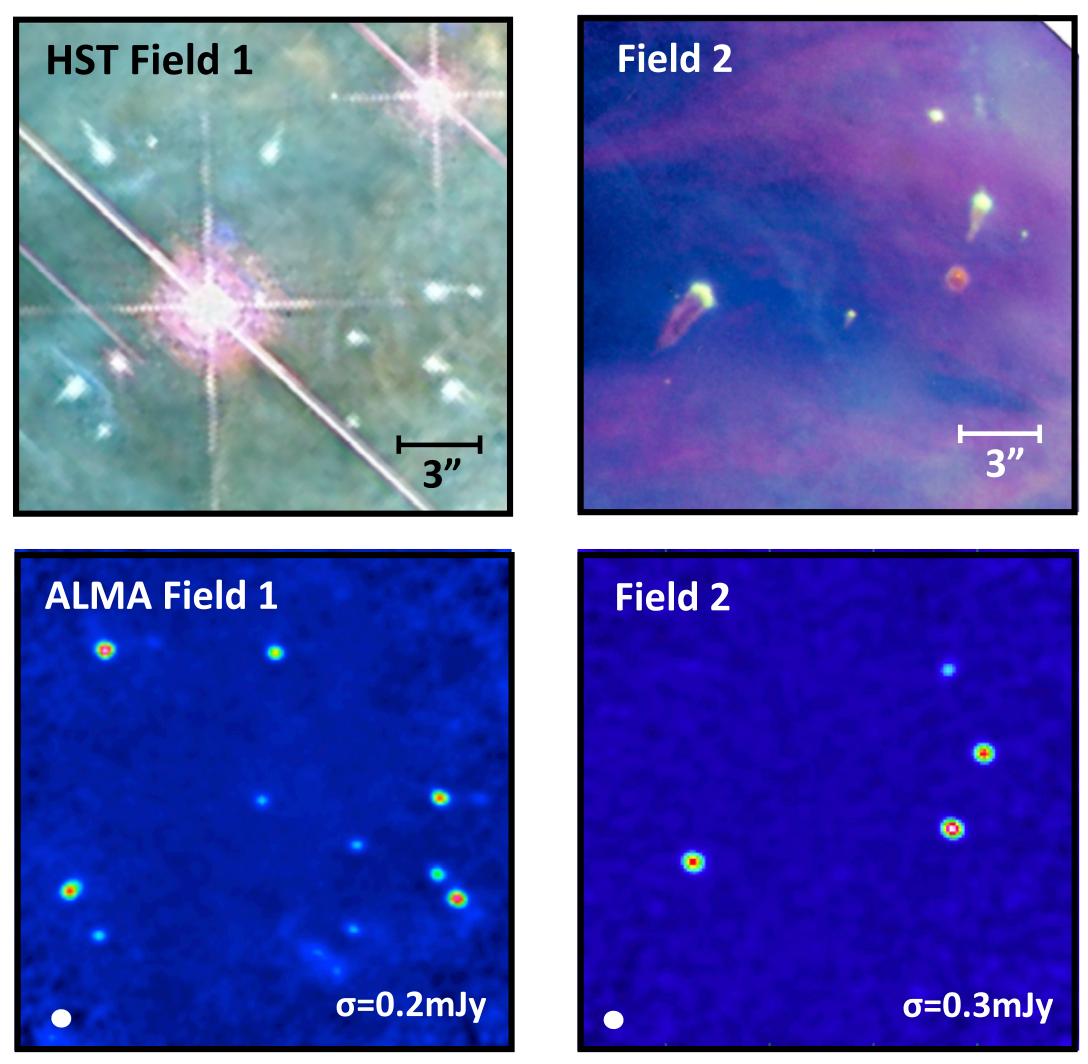

Figure 2. Top: HST images from Bally et al. (2000) of Fields 1 and 2 that were observed with ALMA in Cycle 0. Bottom: corresponding ALMA 0"'5 FWHM resolution $856 \mu \mathrm{m}$ observations. These fields have image sizes of $20^{\prime \prime} \times 20^{\prime \prime}$ and are centered at the positions shown in Table 1 . The ALMA images are the highest resolution observations taken at submillimeter wavelengths of the central regions of the Orion Nebula Cluster.

(A color version of this figure is available in the online journal.) 

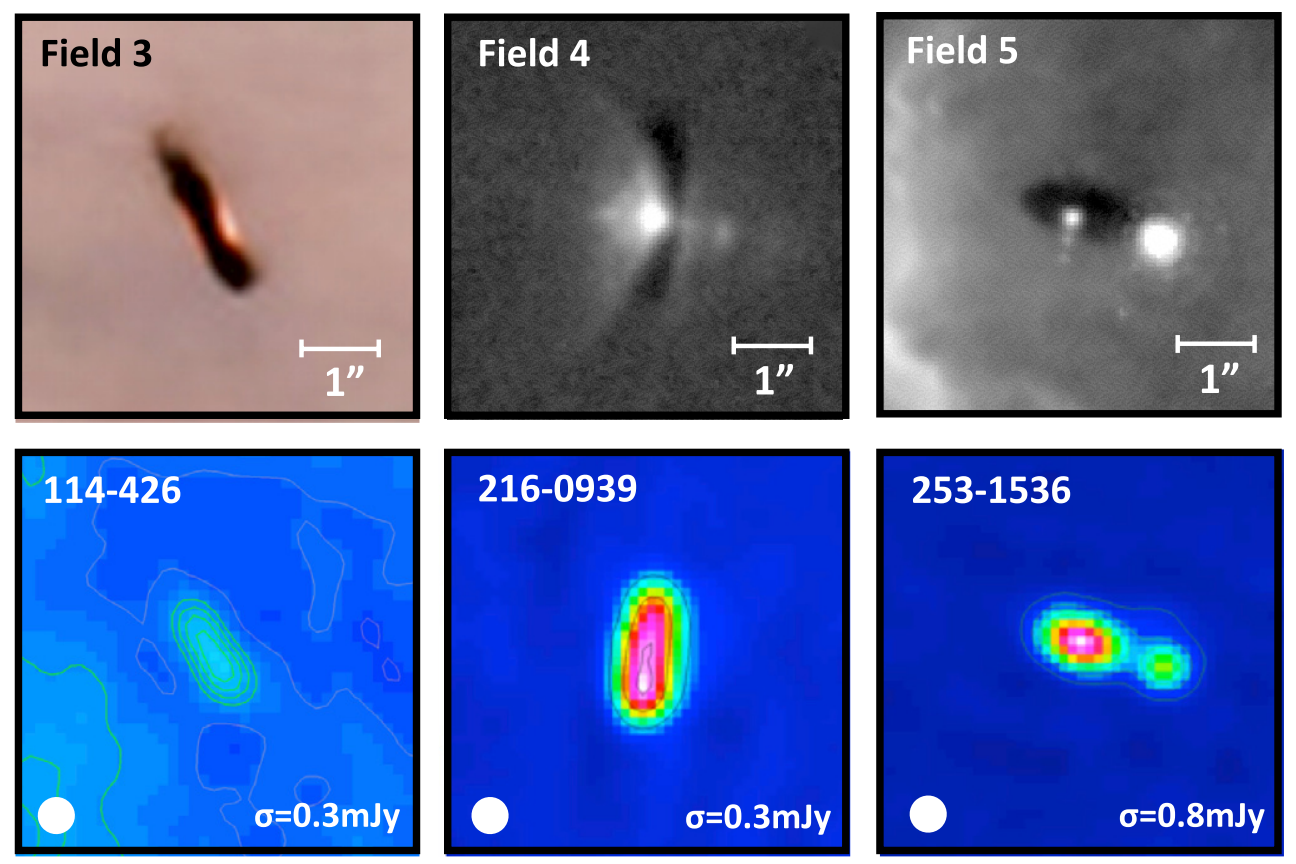

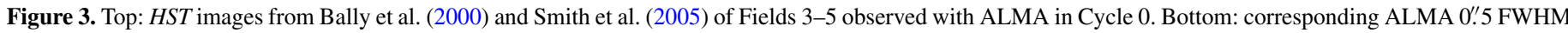
resolution $856 \mu \mathrm{m}$ observations. The image sizes are $5^{\prime \prime} \times 5^{\prime \prime}$.

(A color version of this figure is available in the online journal.)

Table 2

22 Orion Proplyds Targeted in ALMA Early Science Observations

\begin{tabular}{|c|c|c|c|c|c|c|c|c|c|c|}
\hline Field & $\begin{array}{l}\text { Proplyd } \\
\text { Name }\end{array}$ & $\begin{array}{c}\alpha(\mathrm{J} 2000) \\
(\mathrm{h} \mathrm{m} \mathrm{s})\end{array}$ & $\begin{array}{l}\delta(\mathrm{J} 2000) \\
(\mathrm{deg} \mathrm{m} \mathrm{s})\end{array}$ & $\begin{array}{c}F_{856 \mu \mathrm{m}} \\
(\mathrm{mJy})\end{array}$ & $\begin{array}{c}F_{\mathrm{ff}}^{856 \mu \mathrm{m}} \\
(\mathrm{mJy})\end{array}$ & $\begin{array}{l}F_{\text {dust }} \\
(\mathrm{mJy})\end{array}$ & $\begin{array}{l}M_{\text {disk }} \\
\text { (Mjup) }\end{array}$ & $\begin{array}{c}\mathrm{d}\left(\theta^{1} \mathrm{C}\right) \\
(\mathrm{pc})\end{array}$ & $\begin{array}{l}\text { Maj, Min, P.A. } \\
\text { (AU, AU, deg) }\end{array}$ & Notes \\
\hline 1 & $157-323$ & $5: 35: 15.74$ & $-5: 23: 22.49$ & $2.8 \pm 0.3$ & $1.0 \pm 0.1$ & $1.8 \pm 0.2$ & $0.89 \pm 0.12$ & 0.022 & & \\
\hline 1 & $158-323$ & $5: 35: 15.84$ & $-5: 23: 22.47$ & $15.6 \pm 0.3$ & $10.4 \pm 0.9$ & $5.1 \pm 0.9$ & $2.50 \pm 0.46$ & 0.019 & $113,22,+55$ & \\
\hline 1 & $158-326$ & $5: 35: 15.85$ & $-5: 23: 25.56$ & $10.6 \pm 0.3$ & $5.0 \pm 1.6$ & $5.6 \pm 1.5$ & $2.72 \pm 0.74$ & 0.020 & $144,66,+57$ & \\
\hline 1 & $158-327$ & $5: 35: 15.79$ & $-5: 23: 26.56$ & $22.9 \pm 0.4$ & $19.1 \pm 1.4$ & $3.8 \pm 1.4$ & $1.84 \pm 0.70$ & 0.021 & $147,96,+45$ & \\
\hline 1 & $161-314$ & $5: 35: 16.11$ & $-5: 23: 14.06$ & $3.1 \pm 0.6$ & 0 & $3.1 \pm 0.6$ & $1.51 \pm 0.29$ & 0.020 & $272,211,+4$ & New \\
\hline 1 & $161-324$ & $5: 35: 16.07$ & $-5: 23: 24.37$ & $5.3 \pm 0.3$ & $4.7 \pm 0.5$ & $0.6 \pm 0.5$ & $0.30 \pm 0.27$ & 0.013 & $103,36,+83$ & New \\
\hline 1 & $161-328$ & $5: 35: 16.08$ & $-5: 23: 27.80$ & $6.1 \pm 0.3$ & $2.2 \pm 0.1$ & $3.9 \pm 0.3$ & $1.89 \pm 0.17$ & 0.015 & $193,76,+67$ & \\
\hline 1 & $163-317$ & $5: 35: 16.29$ & $-5: 23: 16.55$ & $12.5 \pm 0.3$ & $7.6 \pm 0.1$ & $4.9 \pm 0.3$ & $2.35 \pm 0.15$ & 0.014 & $87,68,+38$ & New \\
\hline 1 & $163-323$ & $5: 35: 16.33$ & $-5: 23: 22.56$ & $6.4 \pm 0.3$ & $3.7 \pm 0.5$ & $2.7 \pm 0.6$ & $1.29 \pm 0.29$ & 0.004 & $154,130,+61$ & \\
\hline 1 & $166-316$ & $5: 35: 16.62$ & $-5: 23: 16.12$ & $3.5 \pm 0.4$ & $2.0 \pm 0.4$ & $1.5 \pm 0.6$ & $0.73 \pm 0.28$ & 0.014 & $215,128,+83$ & New \\
\hline 1 & $167-317$ & $5: 35: 16.75$ & $-5: 23: 16.44$ & $26.0 \pm 0.4$ & $25.4 \pm 1.3$ & $0.6 \pm 1.4$ & $0.30 \pm 0.66$ & 0.015 & $121,118,+64$ & \\
\hline 1 & $168-328$ & $5: 35: 16.77$ & $-5: 23: 28.05$ & $7.1 \pm 0.3$ & $3.1 \pm 0.4$ & $4.0 \pm 0.5$ & $1.91 \pm 0.25$ & 0.013 & $131,92,-65$ & \\
\hline 1 & $168-326$ & $5: 35: 16.85$ & $-5: 23: 26.22$ & $23.5 \pm 0.5$ & $20.3 \pm 2.4$ & $3.2 \pm 2.5$ & $1.53 \pm 1.18$ & 0.012 & $222,118,-42$ & New \\
\hline 2 & $169-338$ & 53516.88 & -52338.10 & $\leqslant 0.8$ & $0.3 \pm 0.0$ & $\leqslant 0.5$ & $\leqslant 0.23$ & 0.032 & & \\
\hline 2 & 170-337 & $5: 35: 16.98$ & $-5: 23: 37.05$ & $23.7 \pm 0.4$ & $8.9 \pm 2.2$ & $14.8 \pm 2.2$ & $7.13 \pm 1.08$ & 0.031 & $86,73,+54$ & \\
\hline 2 & $171-334$ & $5: 35: 17.07$ & $-5: 23: 34.04$ & $9.0 \pm 0.4$ & $3.8 \pm 0.9$ & $5.2 \pm 1.0$ & $2.49 \pm 0.49$ & 0.028 & $\ldots, \ldots, \ldots$ & New \\
\hline 2 & $171-340$ & $5: 35: 17.06$ & $-5: 23: 39.77$ & $33.0 \pm 0.5$ & $0.2 \pm 0.0$ & $32.8 \pm 0.9$ & $15.83 \pm 0.42$ & 0.037 & $102,50,+53$ & \\
\hline 2 & $173-341$ & $5: 35: 17.34$ & $-5: 23: 41.49$ & $2.9 \pm 0.9$ & $1.0 \pm 0.1$ & $1.9 \pm 0.5$ & $0.92 \pm 0.22$ & 0.044 & $574,37,-54^{*}$ & New \\
\hline 2 & $177-341$ & $5: 35: 17.68$ & $-5: 23: 40.98$ & $26.4 \pm 0.5$ & $10.9 \pm 0.9$ & $15.5 \pm 0.9$ & $7.48 \pm 0.45$ & 0.049 & $125,100,+58$ & \\
\hline 3 & $114-426$ & $5: 35: 11.32$ & $-5: 24: 26.52$ & $7.0 \pm 1.2$ & 0 & $7.0 \pm 1.2$ & $3.38 \pm 0.56$ & 0.195 & & New \\
\hline 4 & 216-0939 & $5: 35: 21.58$ & $-5: 09: 38.96$ & $94.9 \pm 1.6$ & 0 & $94.9 \pm 1.6$ & $45.84 \pm 0.77$ & 1.605 & $525,150,-7$ & \\
\hline 5 & $253-1536$ & $5: 35: 25.30$ & $-5: 15: 35.40$ & $162.9 \pm 0.9$ & 0 & $162.9 \pm 0.9$ & $78.66 \pm 0.42$ & 0.942 & $268,95,+72$ & \\
\hline
\end{tabular}

Notes. Column 1: field location; Column 2: proplyd name; Column 3 and 4: phase center coordinates; Column 5: integrated continuum flux density, corrected for ALMA primary beam attenuation, with $1 \sigma$ statistical error; Column 6: extrapolated contribution of free-free emission at $856 \mu \mathrm{m}$ using the highest centimeter flux (see Figure 4); Column 7: derived dust continuum flux density from the disk; Column 8: disk mass from ALMA observations (error does not include uncertainties in the flux scale of $\sim 10 \%$ ); Column 9: projected distance from $\theta^{1}$ Ori C; Column 10: disk size: deconvolved semi-major axis, semi-minor axis, position angle; “...” means that the source is effectively a point source. *Note that the disk size for 173-341 is quite uncertain, given its faintness; Column 11: new detections of submillimeter disk emission. 

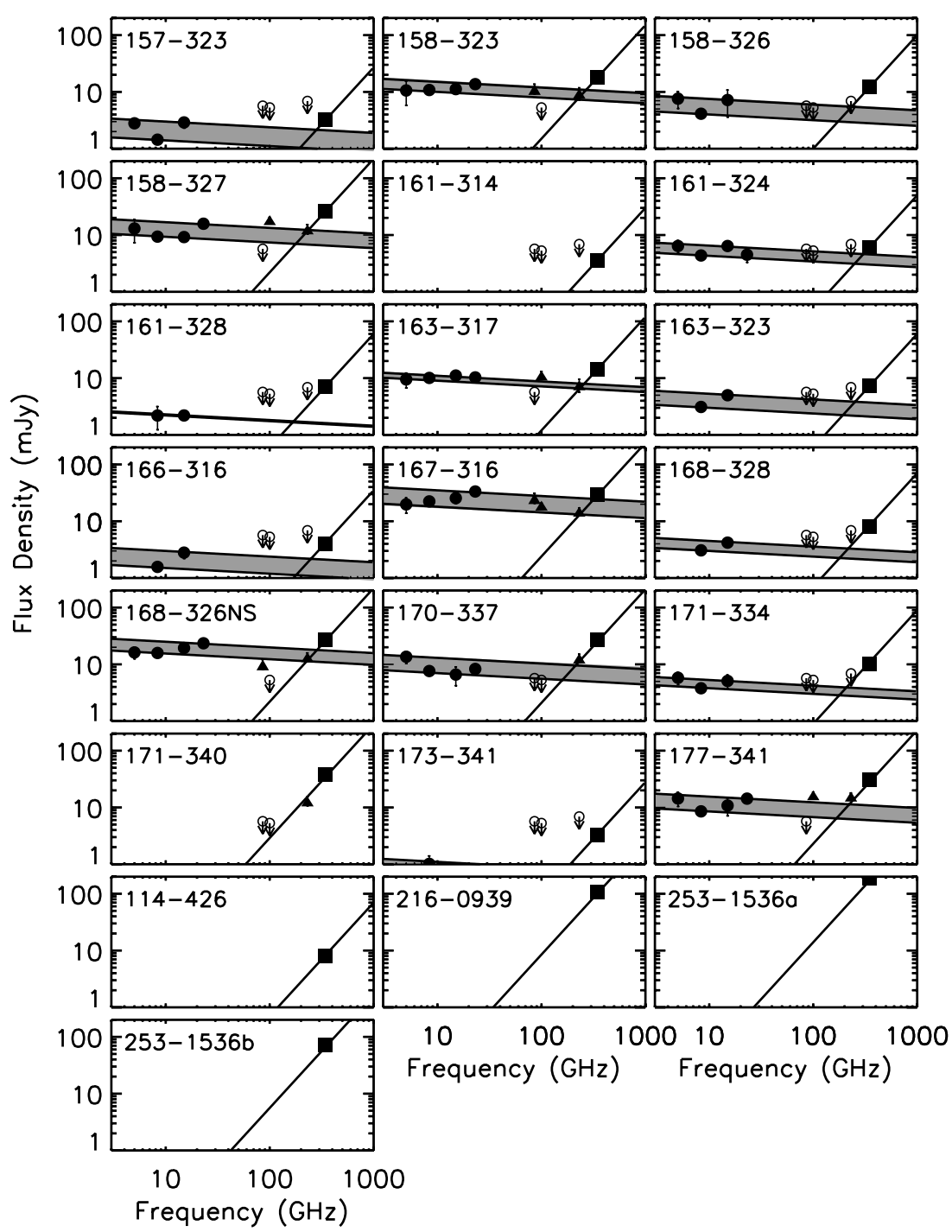

Figure 4. Radio-submillimeter spectral energy distributions of the Orion proplyds detected at $\geqslant 3 \sigma$ with ALMA at $856 \mu \mathrm{m}$. The ALMA measurements are represented by squares, centimeter observations by circles (Garay et al. 1987; Felli et al. 1993b; Zapata et al. 2004), and millimeter observations by triangles (Mundy et al. 1995; Bally et al. 1998b; Eisner \& Carpenter 2006; Eisner et al. 2008). Open circles are upper limits from non-detections and uncertainties not shown are smaller than symbol sizes. The extrapolated range of optically thin free-free emission, $F_{v} \propto v^{-0.1}$, is overlaid in gray. A template to the disk emission, $F_{v} \propto v^{2}$, is shown to guide the eye and reveal the relative contribution of the ionized gas and dust components.

$F_{\mathrm{ff}}^{856 \mu \mathrm{m}}$ is listed in Table 2, and represents the maximum level of free-free emission extrapolated to $856 \mu \mathrm{m}$ from Figure 4 . The highest levels of centimeter emission were used to account for the free-free contributions to result in the most conservative estimate of disk mass. After accounting for the free-free contamination, we estimated $F_{\text {dust }}$ for each source (see Table 2); all 21 of the detected proplyds were detected in thermal dust emission in excess of the free-free emission (see Figure 4).

It is worth noting that we did not correct for background cloud emission as was done for the SMA observations. The SMA synthesized beam size was relatively large in the Mann et al. work (2..5 $1000 \mathrm{AU})$ compared with the ALMA beam $(0 \prime .5 \sim 200 \mathrm{AU})$. The emission probed by the ALMA data is sufficiently compact compared to the beam size that it is not likely to be contaminated severely by background emission. Moreover, the many additional ALMA Cycle 0 baselines provide much better spatial frequency coverage, leading to greater image fidelity and allowing a better separation of the disk emission from the background cloud.
Disk mass estimates and upper limits were then derived from the estimated $F_{\text {dust }}$ values assuming the standard optically thin isothermal relationship (e.g., Beckwith et al. 1990),

$$
M_{\mathrm{disk}}=\frac{F_{\mathrm{dust}} d^{2}}{\kappa_{v} B_{v}(T)}
$$

where $d=400 \mathrm{pc}$ is the distance to Orion (Sandstrom et al. 2007; Menten et al. 2007; Kraus et al. 2007, 2009), $\kappa_{\nu}=$ $0.034 \mathrm{~cm}^{2} \mathrm{~g}^{-1}$ is the Beckwith et al. (1990) dust grain opacity at $856 \mu \mathrm{m}$ with an implicit gas-to-dust mass ratio of 100:1, and $B_{v}(T)$ is the Planck function. We assume a typical disk temperature of $20 \mathrm{~K}$, as in previous disk surveys of Taurus and Ophiuchus by Andrews \& Williams $(2005,2007)$ and the ONC by Mann \& Williams (2009a, 2010), for ease of comparison. Disk continuum emission can deviate from the optically thin limit if the column densities are especially large, an issue that was discussed in detail by Andrews \& Williams (2005). The typical disk structure that could produce the observed $856 \mu \mathrm{m}$ flux densities in Orion would imply the brighter disks could 
have up to $\sim 10 \%-25 \%$ of their emission being optically thick (see Figure 20 of Andrews \& Williams 2005), resulting in an underestimation of their disk masses.

An overall disk mass sensitivity for the survey was determined by measuring the fraction of sources that could be detected at $\geqslant 3 \sigma$ as a function of $M_{\text {disk }}$, depending on the varying levels of free-free emission and target locations within each field. We find that the observed fields are $100 \%$ complete for $M_{\text {disk }} \geqslant 1.2 M_{\text {jup }}\left(1 M_{\text {jup }}=9.5 \times 10^{-4} M_{\odot}\right)$ and $50 \%$ complete for $M_{\text {disk }} \geqslant 0.4 M_{\text {jup }}$. For comparison, the SMA survey at $880 \mu \mathrm{m}$ was $100 \%$ complete for $M_{\text {disk }} \geqslant 8.7 M_{\text {jup }}$ (Mann \& Williams 2010), $7 \times$ higher than the ALMA data.

We were able to determine disk masses for all 21 proplyd detections, as there was sufficient dust emission in excess of the free-free contamination (see Table 2). In addition to the 22 HST-identified proplyds surveyed, we observed 14 sources from the ACS Survey of the HST Treasury Program (Robberto et al. 2013; Ricci et al. 2008), 10 sources from the CTIO/ Blanco $4 \mathrm{~m}$ near-infrared survey by Robberto et al. (2010), and 2 massive stars, $\theta^{1}$ Ori $\mathrm{C}$ and $\theta^{1}$ Ori $\mathrm{F}$ (see Table 3 ). Only two of the non-proplyd sources were detected, including a newly discovered disk around 113-438 and the recovery of the disk around 253-1536b, which was originally discovered through SMA imaging (Mann \& Williams 2009b). The ALMA observations place stringent $(3 \sigma)$ upper limits of $0.1-0.6 M_{\text {jup }}$ on the disk masses for the undetected targets (see Table 3). These disks, if they exist, must be not only low in mass, but are likely smaller than $\sim 0$ '. 15 ( $\sim 60$ AU; Bally et al. 2000; Vicente \& Alves 2005) to be unseen in the HST images. No dust emission was detected toward the massive stars in this survey, $\theta^{1}$ Ori $\mathrm{C}$ (spectral type O6) and $\theta^{1}$ Ori F (spectral type B8). In computing upper limits of $\sim 0.12 M_{\text {jup }}$ for these targets, we adopted a higher dust temperature of $40 \mathrm{~K}$ (see Beuther et al. 2002; Sridharan et al. 2002). The disk-to-stellar mass ratio for these massive stars is $\lesssim 0.12 M_{\text {jup }} / 40 M_{\odot} \sim 3 \times 10^{-6}$, significantly lower than the typical range of $10^{-1}$ to $10^{-3}$ for T Tauri and HAeBe stars, implying that either massive stars do not form with disks or that their disks have much shorter lifetimes (Williams \& Cieza 2011).

The giant silhouette disk 114-426 was detected for the first time at submillimeter wavelengths with these ALMA observations. This disk has been one of the most puzzling objects in Orion, since it is the largest and most prominent optical disk in the entire ONC, but it had never been detected at long wavelengths (Bally et al. 1998b; Eisner \& Carpenter 2006; Eisner et al. 2008; Mann \& Williams 2010). An 1100 AU disk seen nearly edge-on in HST images (see Figure 3), 114-426 is found to have a surprisingly low flux of $7 \mathrm{mJy}$, over an order of magnitude less than the other giant silhouette disk in Orion, 216-0939 ( 95 mJy; see Table 2). The nature of this interesting disk will be the subject of a forthcoming article from our team (J. Bally et al., in preparation).

\subsection{Disk Masses and Distance from $\theta^{1}$ Ori C}

Figure 5 shows the disk masses (and flux densities at $856 \mu \mathrm{m}$ ) in the $\mathrm{ONC}$ as a function of their projected distance from the massive star $\theta^{1}$ Ori $C$, including the previous SMA results from Mann \& Williams (2010) to fill in the intermediate distances not yet probed with ALMA. The $3 \sigma$ upper limits for both surveys are indicated as gray arrows. All of the known proplyds in the central field, i.e., within $9^{\prime \prime}$ of $\theta^{1}$ Ori $\mathrm{C}(\sim 0.02 \mathrm{pc})$, are included. This plot reveals clearly that disk masses tend to be substantially lower when they are located closer to $\theta^{1}$ Ori C.

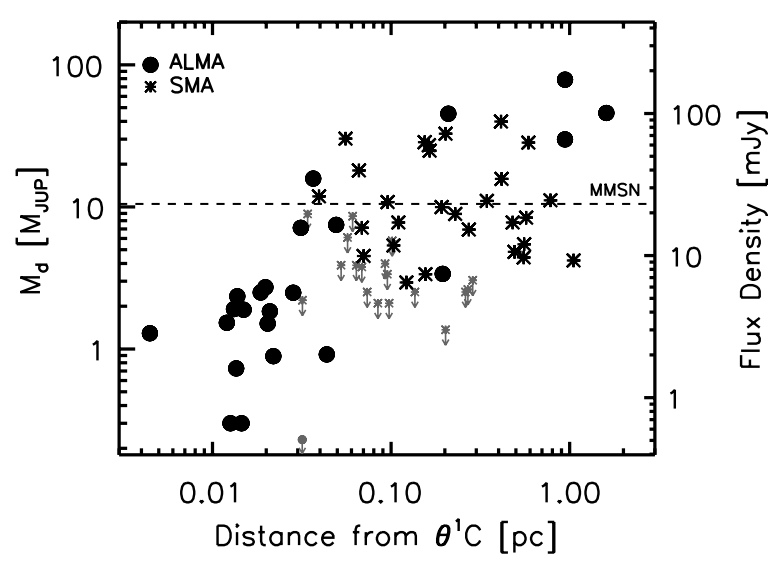

Figure 5. Circumstellar disk masses plotted against their projected distances from the massive O-star, $\theta^{1}$ Ori C. Large black dots represent ALMA detections, while stars represent SMA detections at $880 \mu \mathrm{m}$ for proplyds not yet observed with ALMA. A gray dot and gray stars represent the $3 \sigma$ upper limits for the proplyds not detected in the observed fields. In total, $70 \mathrm{HST}$-identified proplyds surveyed with both the ALMA and the SMA are plotted here. The dashed line represents the MMSN value of $10 M_{\text {jup }}$. The observations expose the trend of decreasing disk masses at smaller separations from $\theta^{1}$ Ori $\mathrm{C}$, particularly within $\sim 0.03 \mathrm{pc}$ of the O-star, where there is a lack of disks more massive than $\sim 3 M_{\text {jup }}$.

Using the correlation tests described by Isobe et al. (1986), including the Cox Hazard Model, Generalized Kendall's Tau, and Spearman's Rho tests, that make use of the combined censored data set quantitatively confirm this trend, where the probability of no correlation between disk mass and projected distance to $\theta^{1}$ Ori $\mathrm{C}$ is $<10^{-4}$ for all three tests. This provides a strong confirmation of the same relationship that was tentatively noted in the previous SMA survey data, particularly for the most massive disks (Mann \& Williams 2010).

\section{DISCUSSION}

We observed the $856 \mu \mathrm{m}$ continuum emission toward 48 young stars in the ONC using ALMA in Cycle 0, including 22 HST-identified proplyds. With an overall $3 \sigma$ survey sensitivity limit of $\sim 1.2 M_{\text {jup }}$, we detected 23 disks $(\sim 48 \%)$, including 9 that had not been detected previously. Aside from the disks around 253-1536b and 113-438 (see Table 3), these detections coincide with the optically discovered disks from HST observations, highlighting the sensitivity of the space telescope to ONC disks due to their contrast with the bright nebular background. The giant silhouette disk 114-426 was detected for the first time, and has a low flux density of $7 \mathrm{mJy}$, and an estimated mass of 3.9 $M_{\text {jup }}$ (this disk will be the subject of a forthcoming article; J. Bally et al., in preparation). Using this ALMA survey and the results of previous observations with the SMA, we find clear, statistically significant evidence for a marked decrease in the $856 \mu \mathrm{m}$ disk luminosities of the Orion proplyds that have smaller projected separations from the massive star $\theta^{1}$ Ori C. In the assumption that the emission is optically thin, and the dust temperature and opacity are the same for all the disks, this implies that the masses of the Orion proplyds decrease for those disks located near $\theta^{1}$ Ori C.

The origins of that latter relationship could potentially be due to projection artifacts, initial conditions, and/or real evolutionary effects. The true separations between the proplyds and $\theta^{1}$ Ori $\mathrm{C}$ are not known, but one can make a probabilistic argument that relates the projected separations to the true ones for an assumed distribution of orbital eccentricities around the 
Table 3

26 Stars Targeted in ALMA Early Science Observations

\begin{tabular}{|c|c|c|c|c|c|c|}
\hline Field & $\begin{array}{l}\text { Proplyd } \\
\text { Name }\end{array}$ & $\begin{array}{c}\alpha(\mathrm{J} 2000) \\
(\mathrm{h} \mathrm{m} \mathrm{s})\end{array}$ & $\begin{array}{l}\delta(\mathrm{J} 2000) \\
(\mathrm{deg} \mathrm{m} \mathrm{s})\end{array}$ & $\begin{array}{c}F_{856 \mu \mathrm{m}} \\
(\mathrm{mJy})\end{array}$ & $\begin{array}{c}M_{\text {disk }} \\
\text { (Mjup) }\end{array}$ & Notes \\
\hline 1 & $157-326$ & $5: 35: 15.73$ & $-5: 23: 25.66$ & $<0.68$ & $<0.33$ & ACS 4210 \\
\hline 1 & $158-318$ & $5: 35: 15.81$ & $-5: 23: 17.51$ & $<0.69$ & $<0.33$ & ACS 4246 \\
\hline 1 & $160-323$ & $5: 35: 15.97$ & $-5: 23: 22.74$ & $<0.61$ & $<0.29$ & ROB 20402 \\
\hline 1 & $161-323$ & $5: 35: 16.10$ & $-5: 23: 23.20$ & $<0.58$ & $<0.28$ & ROB 2489 \\
\hline 1 & $162-319$ & $5: 35: 16.24$ & $-5: 23: 19.13$ & $<0.60$ & $<0.29$ & ACS 4357 \\
\hline 1 & $163-328$ & $5: 35: 16.28$ & $-5: 23: 27.55$ & $<0.60$ & $<0.29$ & ACS 4383 \\
\hline 1 & $164-321$ & $5: 35: 16.37$ & $-5: 23: 21.15$ & $<0.58$ & $<0.28$ & ROB 2197 \\
\hline 1 & $164-325$ & $5: 35: 16.35$ & $-5: 23: 25.34$ & $<0.58$ & $<0.28$ & ROB 20386 \\
\hline 1 & $\theta^{1} \mathrm{C}$ & $5: 35: 16.47$ & $-5: 23: 22.91$ & $<0.58$ & $<0.11$ & O6-type star \\
\hline 1 & $165-320$ & $5: 35: 16.50$ & $-5: 23: 19.76$ & $<0.58$ & $<0.28$ & ACS 4427 \\
\hline 1 & $167-329$ & $5: 35: 16.66$ & $-5: 23: 28.89$ & $<0.65$ & $<0.32$ & ACS 4486 \\
\hline 1 & $\theta^{1} \mathrm{~F}$ & $5: 35: 16.72$ & $-5: 23: 25.20$ & $<0.63$ & $<0.12$ & B8-type star \\
\hline 2 & $168-342$ & $5: 35: 16.84$ & $-5: 23: 42.28$ & $<0.67$ & $<0.32$ & ROB 3063 \\
\hline 2 & $173-337$ & $5: 35: 17.28$ & $-5: 23: 37.20$ & $<0.57$ & $<0.28$ & ACS 4647, \\
\hline 2 & $174-342$ & $5: 35: 17.41$ & $-5: 23: 41.84$ & $<0.58$ & $<0.28$ & ACS 4696 \\
\hline 2 & $178-344$ & $5: 35: 17.79$ & $-5: 23: 44.24$ & $<0.67$ & $<0.32$ & ACS 4831 \\
\hline 2 & $178-343$ & $5: 35: 17.78$ & $-5: 23: 42.63$ & $<0.64$ & $<0.31$ & ACS 4825 \\
\hline 2 & $178-342$ & $5: 35: 17.77$ & $-5: 23: 42.49$ & $<0.64$ & $<0.31$ & ACS 4827 \\
\hline 3 & $117-421$ & $5: 35: 11.65$ & $-5: 24: 21.40$ & $<0.87$ & $<0.42$ & ACS 3388 \\
\hline 3 & $114-416$ & $5: 35: 11.27$ & $-5: 24: 16.46$ & $<0.91$ & $<0.44$ & ROB 4371 \\
\hline 3 & $114-423$ & $5: 35: 11.44$ & $-5: 24: 23.27$ & $<0.82$ & $<0.39$ & ACS 3360 \\
\hline 3 & $111-436$ & $5: 35: 11.14$ & $-5: 24: 36.36$ & $<1.13$ & $<0.55$ & ACS 3335 \\
\hline 3 & $113-438$ & $5: 35: 11.32$ & $-5: 24: 38.22$ & $93.79 \pm 0.31$ & $45.30 \pm 0.15$ & ROBb 18 \\
\hline 4 & $216-0950$ & $5: 35: 21.58$ & $-5: 09: 49.74$ & $<0.95$ & $<0.46$ & ROB 338 \\
\hline 4 & 218-0945 & $5: 35: 21.77$ & $-5: 09: 45.30$ & $<0.89$ & $<0.43$ & ROB 340 \\
\hline 5 & $253-1536 b$ & $5: 35: 25.23$ & $-5: 15: 35.69$ & $61.86 \pm 2.0$ & $29.88 \pm 0.95$ & ROB 6341 \\
\hline
\end{tabular}

Notes. Column 1: field location; Column 2: proplyd name; Column 3 and 4: phase center coordinates (from Ricci et al. 2008); Column 5: $3 \sigma$ dust continuum flux density upper limit; Column 6: disk mass upper limit; Column 7: Notes: ACS sources from Robberto et al. (2013), ROB sources from Robberto et al. (2010), ROBb source from Robberto et al. (2005).

ONC center of mass (cf. Torres 1999). For a uniform eccentricity distribution, the projected and true separations should be commensurate within a factor of $\sim 2$; for a steeper eccentricity distribution, the projected separations represent a more biased tracer of the true values and could be considered lower limits. Such shifts in the abscissae of Figure 5 would not explain the lack of disks around the targets with very close projected separations from $\theta^{1}$ Ori $\mathrm{C}$, nor do they seem likely to be large enough to erase the overall trend (although they indeed may adjust the basic shape). An intrinsic correlation between the masses of disks and their stellar hosts (as found in the Taurus region by Andrews et al. 2013) could account for the observed trend in Figure 5 if the least massive stars are preferentially located near $\theta^{1}$ Ori $\mathrm{C}$. Unfortunately, the nature of the Orion proplyds makes a direct determination of their stellar masses exceedingly difficult. Hillenbrand \& Hartmann (1998) argued that stellar mass segregation in the ONC works in the opposite sense, with the highest mass stars $\left(\geqslant 5 M_{\odot}\right)$ concentrated toward the cluster center. If that were the case, we should have identified an anti-correlation between disk mass and distance from $\theta^{1}$ Ori C, which is clearly not observed. High optical depths could be responsible for such a correlation, if the disks located near $\theta^{1}$ Ori $\mathrm{C}$ are smaller than the distant disks, and most of their emission comes from optically thick regions. However, no correlation has been observed between disk size and distance from $\theta^{1}$ Ori $\mathrm{C}$ (Vicente \& Alves 2005). Furthermore, a small ( $\sim 50 \mathrm{AU}$; the resolution of $H S T$ ), completely optically thick disk would be detectable by our sensitive ALMA observations, with a flux density of $\sim 55 \mathrm{mJy}$ if viewed face-on, and an order of magnitude lower, $\sim 5.5 \mathrm{mJy}$, if viewed nearly edge-on, suggest- ing the submillimeter wave optical depths are not responsible for the observed correlation.

Instead, the evidence suggests that an externally driven disk evolution factor is likely responsible for the behavior in Figure 5. Tidal stripping by stellar encounters is not only too inefficient for substantial disk destruction in the ONC (Scally \& Clarke 2001; Hollenbach et al. 2000), but, as Mann \& Williams (2009b) argued, the conditions required for disk-disk interactions to deplete disk masses (e.g., Olczak et al. 2006) also implicitly involve very high photoevaporation mass-loss rates. Overall, the data suggest that photoevaporative mass-loss driven by the ultraviolet radiation from $\theta^{1}$ Ori $\mathrm{C}$ is the most dominant process responsible for the observed relationship.

Theoretical models of disk photoevaporation indeed predict mass-loss rates that decrease with distance from the irradiation source (Johnstone et al. 1998; Störzer \& Hollenbach 1999; Richling \& Yorke 2000; Scally \& Clarke 2001; Matsuyama et al. 2003; Adams et al. 2004). These models suggest that only low-mass $\left(\lesssim\right.$ few $M_{\text {jup }}$ ) disks should exist within $\sim 0.01-0.03 \mathrm{pc}$ of $\theta^{1}$ Ori $C$ because of the strong extreme-UV (EUV) irradiation at those distances (Johnstone et al. 1998; Störzer \& Hollenbach 1999; Adams et al. 2004). At larger separations, $\sim 0.03-0.3$ pc, less energetic far-UV photons dominate the radiation field, resulting in lower mass-loss rates and thereby preserving more massive disks for up to a few Myr (e.g., Adams et al. 2004).

This predicted behavior is consistent with the observations in the context of Figure 5. There is a clear lack of massive disks $\left(\gtrsim 3 M_{\text {jup }}\right.$ ) within $0.03 \mathrm{pc}$ of $\theta^{1}$ Ori $\mathrm{C}$ where EUV irradiation dominates, whereas we find a wide range of disk masses (similar to what is found in low-mass star formation regions) at larger 
projected separations in the less destructive FUV-dominated regime. Accordingly, the potential to form a planetary system like our own in the EUV-dominated region of the ONC seems unlikely, given the substantially depleted disk masses there. If these nearby disks have not formed planets already, they may be out of luck unless dust grains have grown very large in these disks, to sizes not probed by submillimeter wavelength observations. Resolved, multi-wavelength observations of the Orion proplyds are required to investigate how far planet formation has already progressed in these young disks. It is interesting to note, however, that the fraction of disks with masses that exceed the nominal "Minimum Mass Solar Nebula (MMSN)" model ( $\sim 10 M_{\text {jup }}$; Weidenschilling 1977) in the more distant FUV-dominated region of the ONC is essentially the same as that found in the low-mass star formation environment of Taurus $(\sim 10 \%$; Andrews et al. 2013). ${ }^{9}$ Overall, these observations support the idea that the strength of the local EUV irradiation field has profound environmental consequences on the potential for giant planet formation in the centers of massive star-forming regions.

In ALMA Cycle 1, we expect to observe the disks around 300 stars in the ONC, including 160 HST-identified proplyds. This larger scale study will allow us to survey disks across a range of distances out to $1.6 \mathrm{pc}$ from $\theta^{1}$ Ori $\mathrm{C}$, to probe different conditions in this massive star forming environment and uncover the overall disk fraction and the potential for forming planetary systems like our own.

This paper makes use of the following ALMA data: ADS/ JAO.ALMA\#2011.0.00028.S. ALMA is a partnership of ESO (representing its member states), NSF (USA) and NINS (Japan), together with NRC (Canada) and NSC and ASIAA (Taiwan), in cooperation with the Republic of Chile. The Joint ALMA Observatory is operated by ESO, AUI/NRAO and NAOJ. The National Radio Astronomy Observatory is a facility of the National Science Foundation operated under cooperative agreement by Associated Universities, Inc.

\section{REFERENCES}

Adams, F. C., Hollenbach, D., Laughlin, G., \& Gorti, U. 2004, ApJ, 611,360

Andrews, S. M., Rosenfeld, K. A., Kraus, A. L., \& Wilner, D. J. 2013, ApJ, 771,129

Andrews, S. M., \& Williams, J. P. 2005, ApJ, 631, 1134

Andrews, S. M., \& Williams, J. P. 2007, ApJ, 671, 1800

Bally, J., O’Dell, C. R., \& McCaughrean, M. J. 2000, AJ, 119, 2919

Bally, J., Sutherland, R. S., Devine, D., \& Johnstone, D. 1998a, AJ, 116, 293
Bally, J., Testi, L., Sargent, A., \& Carlstrom, J. 1998b, AJ, 116, 854

Beckwith, S. V. W., Sargent, A. I., Chini, R. S., \& Guesten, R. 1990, AJ, 99, 924

Beuther, H., Schilke, P., Menten, K. M., et al. 2002, ApJ, 566, 945

Butler, B. 2012, ALMA Memo 594

Churchwell, E., Felli, M., Wood, D. O. S., \& Massi, M. 1987, ApJ, 321, 516

Da Rio, N., Robberto, M., Soderblom, D. R., et al. 2009, ApJS, 183, 261

Eisner, J. A., \& Carpenter, J. M. 2006, ApJ, 641, 1162

Eisner, J. A., Plambeck, R. L., Carpenter, J. M., et al. 2008, ApJ, 683, 304

Felli, M., Churchwell, E., Wilson, T. L., \& Taylor, G. B. 1993a, A\&AS, 98, 137

Felli, M., Taylor, G. B., Catarzi, M., Churchwell, E., \& Kurtz, S. 1993b, A\&AS, 101,127

Garay, G., Moran, J. M., \& Reid, M. J. 1987, ApJ, 314, 535

Henney, W. J., \& O’Dell, C. R. 1999, AJ, 118, 2350

Hillenbrand, L. A., \& Hartmann, L. W. 1998, ApJ, 492, 540

Hollenbach, D. J., Yorke, H. W., \& Johnstone, D. 2000, in Protostars and Planets

IV, ed. V. Mannings, A. P. Boss, \& S. S. Russell (Tucson, AZ: Univ. Arizona Press), 401

Hubickyj, O., Bodenheimer, P., \& Lissauer, J. J. 2005, Icar, 179, 415

Isobe, T., Feigelson, E. D., \& Nelson, P. I. 1986, ApJ, 306, 490

Johnstone, D., Hollenbach, D., \& Bally, J. 1998, ApJ, 499, 758

Kraus, S., Balega, Y. Y., Berger, J., et al. 2007, A\&A, 466, 649

Kraus, S., Weigelt, G., Balega, Y. Y., et al. 2009, A\&A, 497, 195

Lada, E. A. 1998, in ASP Conf. Ser. 148, Origins, ed. C. E. Woodward, J. M. Shull, \& H. A. Thronson, Jr. (San Francisco, CA: ASP), 198

Mann, R. K., \& Williams, J. P. 2009a, ApJL, 699, L55

Mann, R. K., \& Williams, J. P. 2009b, ApJL, 694, L36

Mann, R. K., \& Williams, J. P. 2010, ApJ, 725, 430

Matsuyama, I., Johnstone, D., \& Hartmann, L. 2003, ApJ, 582, 893

McCullough, P. R., Fugate, R. Q., Christou, J. C., et al. 1995, ApJ, 438, 394

Menten, K. M., Reid, M. J., Forbrich, J., \& Brunthaler, A. 2007, A\&A, 474,515

Mundy, L. G., Looney, L. W., \& Lada, E. A. 1995, ApJL, 452, L137

O’Dell, C. R., \& Wen, Z. 1994, ApJ, 436, 194

Olczak, C., Pfalzner, S., \& Spurzem, R. 2006, ApJ, 642, 1140

Reggiani, M., Robberto, M., Da Rio, N., et al. 2011, A\&A, 534, A83

Ricci, L., Robberto, M., \& Soderblom, D. R. 2008, AJ, 136, 2136

Richling, S., \& Yorke, H. W. 2000, ApJ, 539, 258

Robberto, M., Beckwith, S. V. W., Panagia, N., et al. 2005, AJ, 129, 1534

Robberto, M., Soderblom, D. R., Bergeron, E., et al. 2013, ApJS, 207, 10

Robberto, M., Soderblom, D. R., Scandariato, G., et al. 2010, AJ, 139, 950

Sandstrom, K. M., Peek, J. E. G., Bower, G. C., Bolatto, A. D., \& Plambeck, R. L. 2007, ApJ, 667, 1161

Scally, A., \& Clarke, C. 2001, MNRAS, 325, 449

Smith, N., Bally, J., Licht, D., \& Walawender, J. 2005, AJ, 129, 382

Sridharan, T. K., Beuther, H., Schilke, P., Menten, K. M., \& Wyrowski, F. 2002, ApJ, 566, 931

Störzer, H., \& Hollenbach, D. 1999, ApJ, 515, 669

Torres, G. 1999, PASP, 111, 169

Vicente, S. M., \& Alves, J. 2005, A\&A, 441, 195

Weidenschilling, S. J. 1977, Ap\&SS, 51, 153

Williams, J. P., Andrews, S. M., \& Wilner, D. J. 2005, ApJ, 634, 495

Williams, J. P., \& Cieza, L. A. 2011, ARA\&A, 49, 67

Zapata, L. A., Rodríguez, L. F., Kurtz, S. E., \& O’Dell, C. R. 2004, AJ, 127,2252

\footnotetext{
9 Although it is worth noting that there are still strong selection effects at play in the currently incomplete ONC disk mass census that will need to be revisited when forthcoming ALMA data sets become available.
} 